\title{
Thank you to our reviewers
}

The BDJ editorial team would like to thank all the people who help maintain the quality of scientific papers in the BDJ by acting as reviewers of the 400 plus manuscripts submitted. The following list contains the referees who have worked on scientific papers during 2007.

Liam Addy

Martin Addy

Irfan Ahmad

Simon Allum

Toshihiro Ansai

Philip Atkin

Paul Averley

Jeremy Bagg

Wendy Baird

Dean Barker

David Bartlett

Mark Bartold

Paul Batchelor

Geoffrey Bateman

Claire Bates

John Beal

William Becker

Heather Beckett

Garmon Bell

P. Benson

Viv Binnie

Karl Bishop

Agnes Bloch-Zupan

Brian Bonner

Stephen Bonsor

Henk Brand

David Brennan

Katherine Brooke-Wavell

Vanita Brookes

Malcolm Bruce

Paul Brunton

Mary Burke

Trevor Burke

Mark Burrell

Lyndon Cabot

Peter Carrotte

Lachlan Carter

Robert Chate

Ivor Chestnutt

Mary Clarke

Peter Cleaton-Jones

John Cottingham

David Craig

Ray Croucher

Roger Currie

Adrian Curtis

Peter Day

Kathryn Derringer

Kelvin Douglas
Martin Downer

Caroline Drugan

Monty Duggal

Christopher Dugmore

Stephen Dunne

Justin Durham

Kenneth Eaton

Janice Ellis

Lester Ellman

Roger Ellwood

Dafydd Evans

Angela Fairclough

Janice Fearne

Michael Fenlon

Janice Fiske

Padhraig Fleming

Jennifer Foley

Ruth Freeman

Martin Fulford

Sabrina Fuller

Jenny Gallagher

Matthew Garnett

Clive Gibson

Nicholas Goodger

Ronald Gorter

Andrew Gould

Robin Gray

Nicholas Grey

Joseph Harper

Rebecca Harris

Jeremy Hayes

Peter Heasman

Stephen Henderson

Ross Hobson

Susan Hooper

Gerry Humphris

Anthony Ireland

Daryll Jagger

Nick Jepson

Waseem Jerjes

Chris Johnston

Andrew Joiner

Asbjorn Jokstad

Clare Jones

Richard Kahan

Paul Keogh

Edwina Kidd

Jules Kieser

Stella Kwan
Stephen Lambert-Humble

Sharon Lee

Peter Leggat

Jason Leitch

Raymond Li

John Lowry

Phil Lumley

Zania Lung

Friederike Luther

Christopher Lynch

lain Mackie

Jimmy Makdissi

Nicholas Malden

Maxwell Graham Manley

Neil Mansfield

John Marley

Michael Martin

Debora Matthews

Bernard McCartan

Robert McCormick

Giles McCracken

Gerald McGarry

Colman McGrath

Grant McIntyre

Niall McLeod

Anthony Mellor

Christopher Mercer

Alan Mighell

Brian Millar

Alex Milosevic

Basil Mizrahi

Claire Morgan

Christopher Morris

Tania Murphy

J. Tim Newton

Kevin O'Brien

Richard G. Oliver

Richard J. Oliver

Chris Orr

Richard Palmer

Menal Pancholi

S. Parekh

Steven Parker

Shanon Patel

Allan Pau

Stephen Porter

Alison Qualtrough

Anand Rao

Patricia Reynolds
Scott Rice

Wayne Richards

Anthony Roberts

Frank Roberts

Graham Roberts

Peter G. Robinson

W. Peter Rock

Elizabeth Roebuck

Mel Rosenberg

Margaret Ross

Jonathan Sandy

Robin Seymour

Jonathan Shepherd

Gillian Smith

Paul Speight

Jimmy Steele

David Stirrups

Jason Stokes

Graham Stuart

Sadie Thomas

Colin Tilley

Jack Toumba

Christopher Tredwin

Cemal Ucer

Sergio Uribe

Basim Uthman

V. Ipe Varghese

Kathryn Waddington

Alison Warburton

Richard Watt

Keith Webster

Richard Welbury

Helen Whelton

D. Anne White

Christine Whitworth

Tony Wildsmith

David Williams

Nairn Wilson

Marcus Woof

Robert Yee

Sin Young

Alix Young

Callum Youngson

Thank you also to anyone who reviewed a manuscript towards the end of this year whose name may not appear above. 